\title{
Herrn dr. Ernst bachmann zum 70. Geburtstag
}

Lieber Kollege Bachmann,

Wenn wir heute an Ihrem 70. Geburtstag Ihrer gedenken, so in erster Linie als eines der Hauptinitianten unserer Zeitschrift. Ihrer Tatkraft verdankt sie ihr rasches Ent-stehen. Ganz besonders freuen wir uns, Sie in jugendlicher Frische und mit jugendlicher Initiative in unserm Redak-tionskomitee mitarbeiten zu sehen, woselbst Sie einen guten Teil der Last tragen, wie Sie dies überall tun, wo Sie ein Amt angenommen haben und es sind deren manche im Dienste der Tuberkulosebekämpfung.

Aber nicht nur Ihr unentwegter Arbeitsgeist macht Sie zu einem so hochgeschätzten Mitarbeiter. Ihre Aufge-schlossenheit überhaupt und besonders für alle Fragen, die die Tuberkulose betreffen, Ihre ganz außerordentliche Er-fahrung auf dem weiten Gesamtgebiet der Tuberkulose, angefangen bei den theoretischen Grundlagen über die Diagno-stik, die Gutachtertätigkeit, das vielschichtige Gebiet der Fürsorge, die Sanatoriumsbehandlung und vor allem auch die Nachbehandlung der Tuberkulösen mit der Wiederein-gliederung der ehemals Kranken in den Arbeitsprozeß veranlaßt uns imnier wieder, bei İhnen Rat und Unter-stützung zu suchen und, was besonders angenehm ist, auch zu finden.

Herr Kollege Bachmann, wir, haben zusammen die nun schon 20 Jahre zurückliegende entscheidende Wandlung der alten Anschauungen über Tuberkulose in die Lehre von den Infiltrat-Tuberkulosen mitgemacht. Wir sind zusammen - es scheint mir erst gestern gewesen zu sein - unter die „Früh-infiltratisten” gegangen in der zweifellos richtigen Erkennt-nis, daß hier ein neuer Weg in der Lehre vom Entstehen tuberkulöser Prozesse sich auftat. Wenn jene Theorie, wie

dies bei jedem neuen Gedanken der Fall ist, große Urn-bildungen erfuhr, die wir aktiv mitarbeitend verfolgten, in unserm Lande so rasch Eingang fand, so haben Sie viel dazu beigetragen. Die Auswirkung der Lehre von den Infiltrat-Tuberkulosen hat sich nicht nur theoretisch, sondern recht bald praktisch, besonders für die Gutachtertätigkeit, für die Indikationsstellung zu Kuren und anderen Behandlungen, als sehr wertvoll erwiesen. Nicht zuletzt führten die neuen Anschauungen zu der vielleicht zunächst etwas unerwarteten Erkenntnis über Häufigkeit und Bedeutung der Primo-infektion der Erwachsenen.

Als Präsident der „Schweizerischen Vereinigung gegen die Tuberkulose” haben Sie neben der großen organisa-torischen Arbeit es stets verstanden, interessante und aktuelle Probleme auf die Tagesordnung zu setzen und zu diskutieren. So haben Sie diese Versammlungen zu überaus wertvollen und recht beliebten Zusammenkünften, zu einer Art Fortbildungszentren gestaltet. Wir haben stets die Auffassung vertreten, daß die gesamte Tuberkulosebekämpfung, die nach Gesetz Aufgabe der Kantone ist, wie bisher, der so gut organisierten privaten Tuberkulosebekämpfung weiter hätte übertragen werden sollen. Die private Tuberkulosebekämpfung verfügt dank Îhrer Initiative über ein ausgezeichnet geschultes Personal, aus dem der Grundstock weiterer Equipen hätte gebildet werden können, ein Personal, das durch langjährige Er-fahrung über die Eigentümlichkeiten der Krankheit und besonders auch über die Psychologie der Kranken und ihrer Angehörigen so gut orientiert ist, sodaß es auch 
größere Aufgaben der Depistage und Versorgung ohne Schwierig-keiten hätte übernehmen und bewältigen können. Nun, die ,,Staatsmedizin” will sich jetzt selbst direkt an den Koloß Tuberkulose wagen, allerdings nachdem ihm dieTuberkulose-Fürsorge in unserm Lande während Jahrzehnten manchen Schlag versetzt und ihm vor allem die Lebensbedingungen langsam, aber stetig und sicher immer mehr abgeschnitten hat, nicht zuletzt auch durch das Instrument des von Ihnen seinerzeit mit so viel Nachdruck empfohlenen Tuberkulose-gesetzes von 1928.

Von İhrer Tätigkeit als Präsident der Stiftung Zürcher Heîlstätten Wald und Clavadel sei nur kurz erwähnt, daß Sie auch hier in zäher Arbeit Hervorragendes geleistet haben, zuletzt in der Verwirkl-ichung des schönen Neubaus in Wald, auch hier gegen manche Wïderstände ankämpfend, unentwegt dem gesteckten Ziele zustrebend.

Nachdem so durch persönliche, uneigennützige Organi-sationsarbeit ein weitgehender Ausbau des Sanatoriums-wesens auf privater Grundlage geschaffen worden ist, möchte auch hier die Staatsmaschine der privaten Initiative auf diesem wichtigen Gebiet der Heilkunde den Dank der Republik erstatten. Es mag einen gewissen Trost bedeuten, daß die Tendenzen der Verstaatlichung die Unentbehrlich-keit der weitsichtig geplanten und geschaffenen privaten Institutionen beweisen.

Die Planung und Gründung der vorbildlichen Arbeíts-heílstätte Appisberg war neben den vielen Obliegenheiten eines Präsidenten der Kantonalen Liga zur Bekämpfung der Tuberkulose eines Ihrer persönlichsten Werke. Damit haben Sie das letzte Ziel der Tuberkulosebekämpfung erstrebt, nicht nur die anatomische Heilung zu erreichen, sondern die Wiedereingliederung des einstigen Kranken in den Arbeitsprozeß, ein Problem, über dessen Vielgestaltig-keit und Schwierigkeit der Fachmann nur allzu reichliche Erfahrung hat, während dem Laien so gut wie nichts darüber bekannt ist und er sich daher durch alle mòglichen, bis-weilen so hochtönenden Schlagworte vom Wesentlichen dieses sozial so wichtigen Problems ablenken läßt.

Wenn wir Ihnen an dieser Stelle für all' die geleistete Arbeit, für alle Anregungen und Förderungen den herzlichen Dank sagen, so freuen wir uns, daß Sie die Mitarbeit an unserer Zeítschríft unentwegt fortsetzen und hoffen, daß wir noch lange Zeit darauf zählen dürfen. In Freundschaft und Dankbarkeit Ihr W. Löffler. 